\title{
A Comparison of Vibration based Bearing Fault Diagnostic Methods
}

\author{
Ted Ooijevaar ${ }^{1}$, Kurt Pichler ${ }^{2}$, Yuan $\mathrm{Di}^{3}$ and Clemens Hesch ${ }^{4}$ \\ ${ }^{1}$ DecisionS, Flanders Make (FM), Gaston Geenslaan 8, 3001 Leuven, Belgium \\ ted.ooijevaar@flandersmake.be \\ ${ }^{2,4}$ Linz Center of Mechatronics (LCM), Altenberger Straße 69, 4040 Linz, Austria \\ Kurt.Pichler@lcm.at \\ Clemens.Hesch@lcm.at \\ ${ }^{3}$ Center for Intelligent Maintenance Systems (IMS), University of Cincinnati, 560 Baldwin, Cincinnati, OH 45221-0072, USA \\ diyn@mail.uc.edu
}

\begin{abstract}
This paper presents a benchmark study in which three vibration based bearing diagnostic algorithms are compared. The three methods are a data driven approach developed by the Linz Center of Mechatronics (LCM), an physics based method of Flanders Make (FM) and an approach developed by the Center for Intelligent Maintenance Systems (IMS). Two experimental tests have been performed, an accelerated life time test to degrade a bearing and introduce an operational bearing fault and a gearbox test containing various faulty test bearings. The methods are compared based on their diagnostic performance, practical applicability, training and configuration requirements. Based on the accelerated life time test, it is concluded that the method of IMS and FM, employing bearing specific features, showed to be more sensitive for early bearing fault detection than purely statistical features used in the method of LCM. On the contrary, the method of LCM does not require specific system knowledge and is not limited to bearing monitoring only. The method is more widely applicable to fault monitoring problems. The methods of IMS and LCM seem to outperform the method of FM in the gearbox test. However, the training and testing data used by those methods were acquired for the same bearing sample and for the same bearing assembly. This could lead to a high correlation between the training and testing data and hence a misleading classification accuracy. Therefore, attention should be paid to the quality of the training data. It is concluded that the training data should comprise all relevant system variations, including e.g. remounting of the bearing,

\footnotetext{
Ted Ooijevaar et al. This is an open-access article distributed under the terms of the Creative Commons Attribution 3.0 United States License, which permits unrestricted use, distribution, and reproduction in any medium, provided the original author and source are credited.
}

to ensure that the classification is uniquely based on bearing fault related effects. The methods of IMS and LCM require validated training data of both healthy and faulty bearing scenarios, whereas the method of FM relies on training data of healthy bearings only. In practice, the availability of training data of faulty bearings is often scarce and could make the adoption more complicated. The findings presented in this paper serve as a guideline to support the selection of an appropriate method for practical applications.

\section{INTRODUCTION}

Manufacturing companies continuously try to increase their productivity, among others by avoiding machine down times. The latter involves considerable costs because of the resulting loss of turnover. Monitoring the condition of, for instance, bearings and gears, plays a vital role in the maintenance program of rotating machines. Early fault detection could allow to move from a time based preventive maintenance program to a condition based predictive maintenance strategy and reduce unexpected machine downtime and cost.

Vibration based condition monitoring is an established approach that has been employed by industries for many years in their maintenance program. However, up to this day, machine operators often still base their maintenance decisions on data from the periodical and manual inspection of one single machine, which does not always result in correct conclusions. The common practice is that vibration measurements are periodically recorded using portable vibration sensors and measurement signals are analyzed by an expert to interpret the machine's health condition. This approach can, however, lead to serious misinterpretation, where rapidly growing faults could be missed. 
A continuous condition monitoring approach enables an early detection of machine faults. In this way, the machine condition is continuously tracked and total failures can be anticipated in advance, hence allowing appropriate maintenance actions. Despite its advantages, continuous monitoring program is however still not well adopted by industry. Firstly, because it often involves a high investment cost. Although recent advancements in sensor, acquisition and processing hardware have demonstrated cost-effective solutions (Albarbar, Mekid, Starr, \& Pietruszkiewicz, 2008; Ompusunggu, Ooijevaar, Kilundu, \& Devos, 2018), the economical benefit of the investment is still not clear and hard to quantify. Secondly, because many of those systems still require an expert to interpret the analysis results or are only partly automated. Finally, also because it is not straight forward to select the most appropriate method for a specific application.

Taking into account the bearing monitoring and analysis, a wide range of vibration based bearing fault detection methods have been proposed in literature (Henriquez, Alonso, Ferrer, \& Travieso, 2014; Sait \& Sharaf-Eldeen, 2011; D. Wang, Tsui, \& Mia, 2017). Approaches that utilize time domain features (e.g. crest factor, kurtosis), frequency and cepstral domain features (e.g. envelope analysis, cepstral coefficients) usually assume stationary machine conditions. Other methods such as cyclo-stationary analysis (i.e. second order technique in the frequency domain) and time-frequency domain analysis (e.g. Wigner-Ville distribution, Hilbert-Huang transform and wavelet transform based features) are more appropriate for non-stationary processes. Some of those methods are purely data driven, whereas others use the physical relation between the bearing geometry, the rotational shaft speed and the bearing specific fault frequencies associated to the impulse behavior introduced by bearing faults.

Once fault sensitive features have been extracted, learning approaches (i.e. supervised, semi-supervised or unsupervised) can be used to distinguish between healthy and faulty states. The machine should be forced to work in different conditions, including all faulty states, to train a supervised machine learning model. Alternatively, semi-supervised anomaly detection methods only require data from normal conditions, and hence do not need data of a faulty machine. However, anomaly detection can only distinguish between normal and faulty conditions. Noise and changing environments should be taken into account to avoid incorrect interpretations.

Typically, it is difficult to objectively compare the performance and applicability of all those diagnostic methods. The type of machine, the type of faults, the fault severity or the operational conditions are often different between studies. Therefore, we have performed a benchmark study to compare the performance of three state-of-the-art condition monitoring methods for bearing fault diagnostics. This study aims to gain knowledge about the applicability, strengths and weaknesses of each individual approach. The findings presented in this paper can serve as a guideline for the selection of an appropriate method for practical applications.

Three methods are considered in this study: a purely data driven feature extraction and classification method developed by Linz Center of Mechatronics (LCM), a physical model based diagnostic method of Flanders Make (FM) and an approach developed by the Center for Intelligent Maintenance Systems of the University of Cincinnati (IMS) utilizing bearing specific fault features in combination with supervised learning approach based on self-organizing maps.

This paper starts with a detailed description of the three diagnostic algorithms that are evaluated in this study. Two different experimental tests, i.e. an accelerated life time test of a ball bearing and a gearbox test containing various bearing faults, have been performed and are described in section 3 . This is followed by a detailed description of the results that have been obtained by each method. In section 5 the results are compared and discussed in detail. The paper is finalized with a set of guidelines on the applicability of the three methods for machine condition monitoring.

\section{DESCRIPTION OF THE BEARING FAULT DIAGNOSTIC ALGORITHMS}

\subsection{Method LCM}

The fault diagnosis approach adopted here is purely data driven, i.e. it incorporates no physical knowledge about the monitored system. That makes it on one hand more flexible and applicable to many kinds of systems, machines or components. On the other hand, incorporating extra knowledge usually improves the diagnostic ability of a condition monitoring system.

In a first step, a big number of features is extracted from the vibration signals. Feature extraction for vibration analysis has been discussed in numerous publications, extensive reviews can be found for instance in (D. Wang et al., 2017; Singh \& Vishwakarma, 2015). The extraction of typical statistical features in time domain is described in (Sharma \& Parey, 2016; Lei, He, Zi, \& Hu, 2007; Shen, Wang, Kong, \& Tse, 2013; Decker \& Lewicki, 2003; Alattas \& Basaleem, 2007; Boldt, Rauber, \& Varejão, 2013; Jalil, Butt, \& Malik, 2013; Suma \& Gurumurthy, 2010; Kollialil, Gopan, Harsha, \& Joseph, 2013). Features in time-frequency and frequency domain are proposed and investigated in (Sharma \& Parey, 2016; Lei et al., 2007; Alattas \& Basaleem, 2007; Boldt et al., 2013). Typical symptom parameters in frequency domain for rotating machinery are extracted in $(\mathrm{H}$. Wang \& Chen, 2007). Adopting the spectral kurtosis for vibration monitoring is examined in (Rao, 2015; Antoni \& Randall, 2006). In (McClintic, Lebold, Maynard, Byington, \& Campbell, 2000; Assaad, Eltabach, \& Antoni, 2014) features of residual and 
difference signal are extracted by using for instance autoregressive models. Features in wavelet domain are introduced in (Heidari Bafroui \& Ohadi, 2014; Jafarizadeh, Hassannejad, Ettefagh, \& Chitsaz, 2008; Bajric, Zuber, Skrimpas, \& Mijatovic, 2016; Kollialil et al., 2013). (Satyam, Sudhakara, \& Devy, 1994; Konstantin-Hansen \& Herlufsen, 2010) examine vibration analysis in cepstral domain. The application of synchronous time averaging is demonstrated for instance in (McFadden \& Toozhy, 2000). We implemented a broad selection of the proposed features to analyze the bearing data. Overall, 83 features were extracted.

In the next step of the supervised learning approach, the dimensionality of the feature space is reduced to avoid the curse of dimensionality. Therefore, the significant features are identified by feature selection procedures as described in (Guyon \& Elisseeff, 2003). In particular, a standard forward selection filter algorithm selecting one feature per step was applied. As selection criterion in each step we use robust distance measures like Dy-Brodley measure (Dy \& Brodley, 2004) or Mahalanobis distance (McLachlan, 1999). Feature selection is stopped when the relative gain of the selection criterion falls below $1 \%$. Both distance measures selected the same features in the first few steps of forward selection until the stopping criterion was reached. Amongst the finally selected features were the short-time energy (Jalil et al., 2013), average magnitude difference (Suma \& Gurumurthy, 2010), interquartile-range (Kollialil et al., 2013), symptom parameters in frequency domain (H. Wang \& Chen, 2007) and energy values for certain wavelet bands (Heidari Bafroui \& Ohadi, 2014).

Once the features are extracted and selected, a classifier in the feature space is trained. For that purpose we use a linear or quadratic normal Bayes classifier (de Ridder et al., 2017). This classifier assumes normally distributed classes and applies Bayes decision rule to those classes. In the linear case, the co-variances of the classes are assumed to be equal. The validity of using normally distributed classifiers was checked by normal probability plots of the feature vectors.

The supervised learning approach implies of course that the method depends on having a sufficient amount of annotated training data for all states (failure modes) to be monitored. There are also classifiers for one-class classification (also referred to as novelty detection) available (Tax, 2001). However, those techniques detect only deviation from a nominal state, without giving any conclusion about the type of the fault. Furthermore, the feature selection process depends on having an annotated data set.

The process of evaluating a new data sample is straightforward: the selected features are extracted from the raw signals, and the classifier is applied to those features. Since many classifiers are able to deliver class membership probabilities, it is generally possible to determine instances lying between

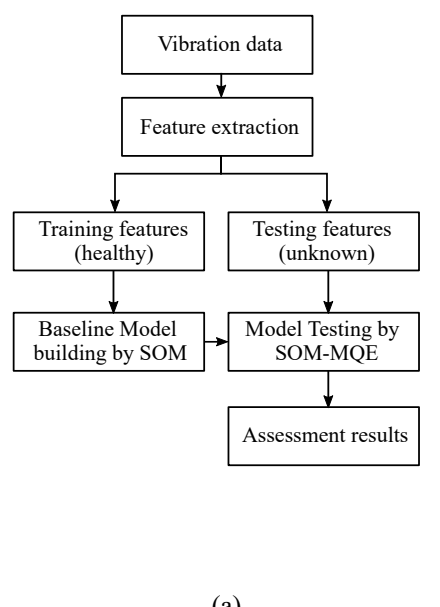

(a)

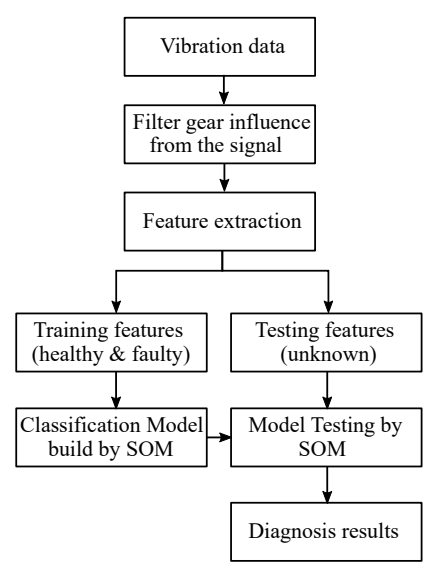

(b)
Figure 1. Framework of IMS's bearing health monitoring approaches: (a) health assessment; (b) fault diagnosis

two distinct states. However, here we restrict the evaluation to crisp class decisions.

Given the data, the whole process of feature extraction, feature selection and classification can be fully automated. The more useful information the training data contain, the better the resulting feature subset and classifier will be. In this context, information means different states, rotation speed, repeated measurements with different samples of the same bearing type, and so on.

\subsection{Method IMS}

The framework of the bearing monitoring approaches are illustrated in Figure 1. Figure 1 (a) shows the method for bearing health assessment while the fault diagnosis flowchart is depicted in Figure 1 (b). The measured vibration data will go through two major steps, which are the feature extraction and the model training/testing. The monitoring results are obtained by the model testing. Both the system domain knowledge and the data-driven techniques will be applied in these steps.

Both approaches share the same feature extraction step, which processes the raw signal and extracts representations which indicate incipient defects of the bearing. There are two types of features that are extracted. Firstly, we extract these features which represent the whole vibration level of the signal since the fault will bring extra vibrations to the system. They contain summaries of statistics, such as kurtosis and root mean squares (RMS), and the energy variations in frequency domain (spectral kurtosis) (Jia, Zhao, Di, Jin, \& Lee, 2017; Jia, Zhao, Di, Li, \& Lee, 2018). The second group of features come from the bearing signatures (i.e. BPFI, BPFO, FTF, BSF, BDF), which are amplitudes at specific bearing signature frequencies (Randall \& Antoni, 2011). In practice, however, these signatures might be disturbed by other com- 
ponents including gears and shafts. Thus, an advanced signal processing technique is applied to isolate such bearing related information from the signal. Through a time synchronous averaging method (Bechhoefer \& Kingsley, 2009; Jia et al., 2017), a spectrum unifying approach (Sawalhi, Randall, \& Forrester, 2014), and the Hilbert Transform, shaft rotating related components are filtered and the bearing signature components are demodulated. Applying FFT on the filtered signal, the bearing specific features are then extracted. Finally, the features used for both approaches include kurtosis, RMS, RMS after spectrual kurtosis filtering, kurtosis after spectral kurtosis filtering, BPFI, BPFO, FTF, BSF, and BDF.

In the second step, a machine learning model is constructed based on the extracted features in each approach respectively. In health assessment, a clustering or distribution estimation method is applied to recognize the normal behavior as the baseline. The monitoring data are then compared with the baseline and the distance, which can be denoted as the assessment score, is utilized to estimate the current health state. The larger the score indicates the more severe the degradation. In fault diagnosis, a classification model is applied to memorize the faulty patterns with various bearing failure modes. In this comparative study, self-organizing map (SOM) (Kohonen, 1998) is utilized to achieve the model training and testing. For bearing health assessment, a SOM model is built by the healthy feature sets and the assessment score is calculated by the minimum quantization error (MQE) (Di et al., 2018). For fault diagnosis, the SOM model is trained by both the normal data and faulty examples and the diagnosis is performed by the evaluate the corresponding best matching unit (BMU) of the testing feature.

Once the model has been constructed, the monitoring process can be employed automatically. An assessment score, or a possible bearing failure mode, is generated as the final result by inputting the vibration measurement. The monitoring prerequisites include the rotating speed of the bearing shaft, the bearing geometric information, the sampling rate of the signal, healthy data (health assessment) and faulty examples (diagnosis).

\subsection{Method FM}

The number of potential faults in bearings is diverse and ranges from surface fatigue to lubrication-related faults. A large part of the bearing faults occur locally and result in periodic impulsive vibrations (Halme \& Andersson, 2009). Other vibration sources in machines such as gears, shafts and noise can mask the effects of bearing faults. The algorithm employed is used to isolate the desired bearing fault-related information from the vibration signal and is summarized in Figure 2.

In the first step the raw vibration signal is angularly re-sampled if the shaft speed variation is significant. This step is not needed when the shaft speed is stationary. Vibrations caused

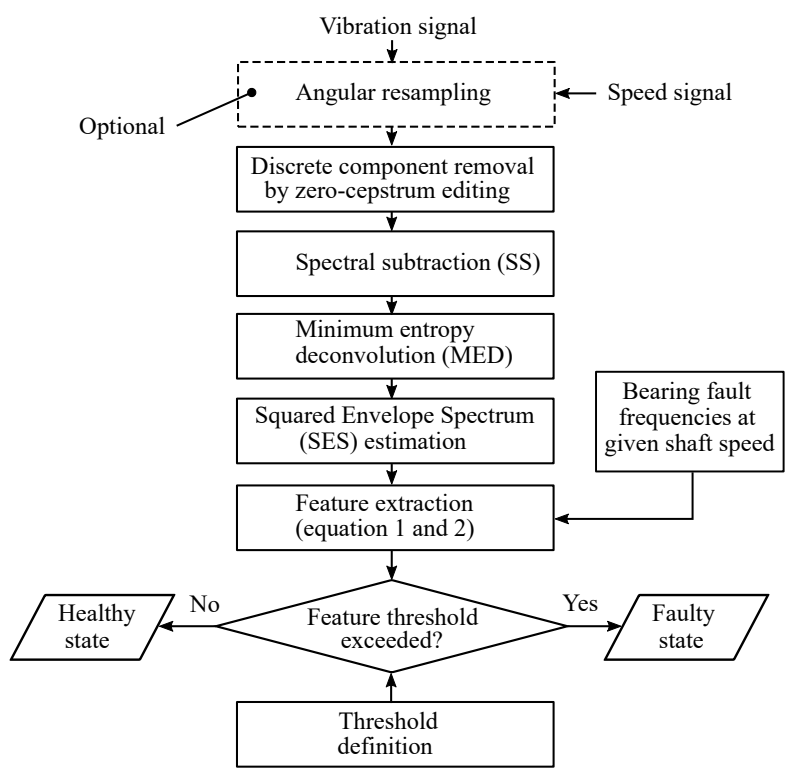

Figure 2. Overview of Flanders Make's vibration based bearing fault detection algorithm.

by gears, screws, shafts are often dominant in the vibration signal of rotating machines. These vibrations are removed from the raw signal in the second step by the zero-cepstrum editing method described in (Barbini, Ompusunggu, Hillis, du Bois, \& Bartic, 2017; Borghesani, Pennacchi, Randall, Sawalhi, \& Ricci, 2013). The filtered signal containing bearingrelated information is then subsequently enhanced by the spectral subtraction and minimum entropy deconvolution (MED). The spectral subtraction is crucial in suppressing random noise, while the MED filtering aims at mainly reducing the transfer path effect such that the impulsiveness of the bearing faultrelated signal is further enhanced if present. The combined processing steps are described in detail in (Ompusunggu et al., 2018). The enhanced signal is used to calculate the squared envelope signal. The spectrum of this envelope signal, which is called squared envelope spectrum (SES), is then estimated (Randall \& Antoni, 2011).

Features corresponding to different bearing faults (e.g. excessive clearance, inner-race fault, outer-race fault, rollingelement fault, cage fault) are extracted from the SES. The bearing fault frequency characteristics (i.e. BPFI, BPFO, BDF, FTF) at given shaft speed must be known. The bearing fault feature $F$ is defined as the summation of the normalized SES magnitude $\overline{S E S}$ of the first four harmonics of a bearing fault frequency of interest $v_{b}$ :

$$
F=\sum_{k=1}^{4} \overline{S E S}\left[k v_{b}\right]
$$

The normalized $\overline{S E S}$ magnitude SES is expressed in the fol- 
lowing equation:

$$
\overline{S E S}[k]=\frac{S E S[k]}{S E S[0]}
$$

with $S E S[0]$ denoting the magnitude of SES at frequency zero.

Finally, a diagnostic decision is made automatically by comparing the feature value with a predetermined threshold. The threshold is defined by a statistical approach using only healthy vibration data, i.e. no fault-state data is required for training. According to (Jablonski, Barszcz, Bielecka, \& Breuhaus, 2013), vibration data can be well fitted by a generalized extreme value distribution due to their one-sided distribution, with probability distribution function $y$ :

$$
\begin{array}{r}
y=\frac{1}{\sigma} t(x)^{k+1} e^{-t(x)} \\
\text { with } t(x)=\left(1+k\left(\frac{x-\mu}{\sigma}\right)\right)^{-1 / k}
\end{array}
$$

This distribution has 3 parameters: shape parameter $k$, scale parameter $\sigma$, location parameter $\mu$. The threshold is set using a high percentile value of the fitted cumulative probability density of the healthy values. In this paper the $96 \%$ value was used as a baseline. The warning threshold is set $3 \mathrm{db}$ above this baseline. The alarm threshold is set $6 \mathrm{db}$ above the baseline (i.e. double of the baseline value) to minimize potential false positive alarms. The bearing is reported to be healthy if the feature value is smaller than the alarm threshold, while a bearing is classified as faulty if the feature value is larger than the alarm threshold.

The entire signal processing and analysis is performed automatically. The information that is required in the algorithm is limited to (1) the shaft rotational speed, (2) the bearing type or geometry to calculate the bearing fault frequencies at the given speed, (3) acceleration data of a healthy bearing for training and (4) acceleration data for testing.

\section{EXPERIMENTAL SET-UPS AND TESTS}

Two types of experiments have been performed: (i) an accelerated life time test (ALT) of a ball bearing on a single shaft drive train set-up and (ii) a test on a more complex gearbox set-up including bearings with various faults. Both tests are described in the next two sections.

\subsection{Accelerate life time test}

The accelerated life time test allows to create an operational fault in a bearing. This test differentiates from other studies on the fact that they are often limited to artificially induced faults. Moreover, the fact that the fault evolution and accumulation can be monitored during the accelerated life time. The experimental set-up used to perform the accelerated life

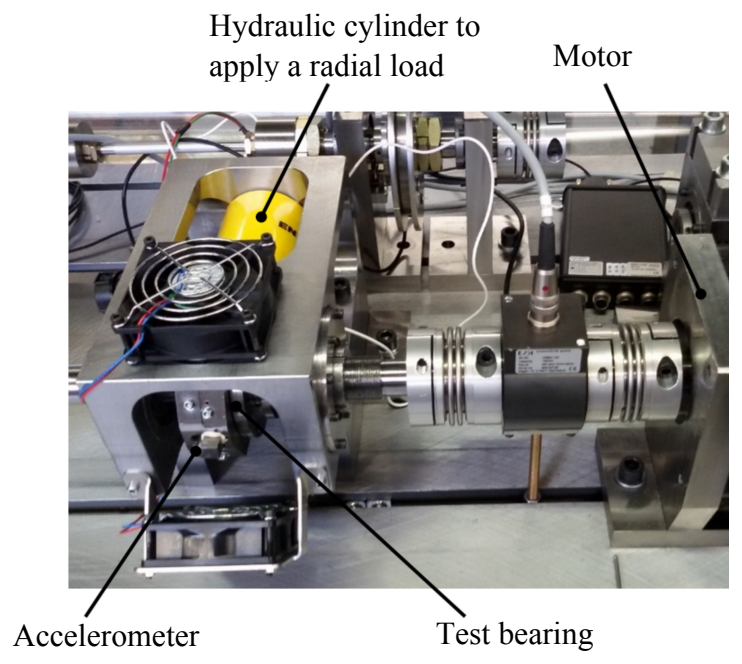

Figure 3. The drive train set-up used to reduce the life time of a bearing to less than a day, allowing to generate vibration data during the accumulation of a operational bearing fault.

time test is shown in Figure 3. The set-up comprises of a single shaft with a test bearing. The shaft is supported with the help of a support bearing on each side. A hydraulic cylinder is used to apply a radial load to the test bearing up to a maximum of $10 \mathrm{kN}$. The test bearing is oil lubricated by an internal oil bath. Two air fans were installed to cool the set-up and avoid overheating of the bearing. The set-up is driven by a motor at a fixed rotation speed of $1500 \mathrm{rpm}$.

The test procedure is schematically illustrated in Figure 4. Vibration measurements were performed under a nominal radial load of $1.5 \mathrm{kN}$ (i.e. $10 \%$ of the dynamic load rating). The radial load was temporarily increased to $9.0 \mathrm{kN}$ (i.e. $65 \%$ of the dynamic load rating) to accelerate the life time of the bearing. In the beginning the interval was 20 minutes, but this has been reduced as soon as the first indication of an incipient fault was noticed in the measured vibration responses. In total $30 \mathrm{vi}-$ bration measurements were performed at the nominal $1.5 \mathrm{kN}$ loading condition and 29 vibration measurements at the high $9.0 \mathrm{kN}$ radial load. The accelerated life time test was stopped

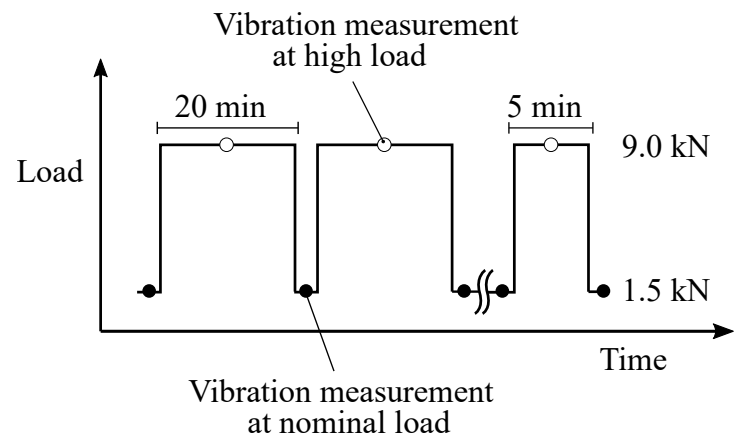

Figure 4. The load was temporarily increased from $1.5 \mathrm{kN}$ to $9 \mathrm{kN}$ to accelerate the life time of the bearing. 


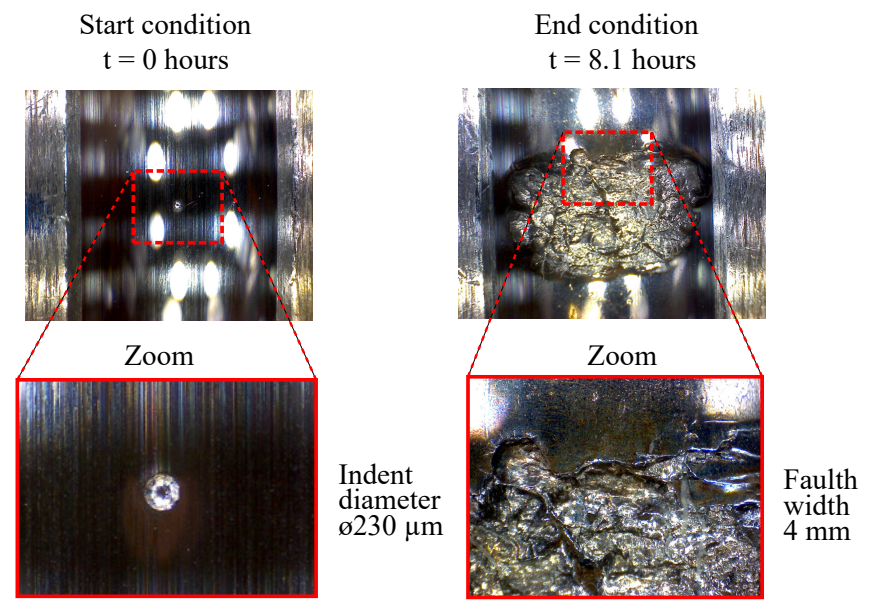

Figure 5. The indentation at the bearing inner race used as the start condition and the surface fatigue fault at the inner race introduced by the accelerated life time test.

when a vibration peak level of $+/-50 \mathrm{~g}$ was reached.

The applied radial load, the radial vibrations in the loading direction and the temperature of the bearing housing were measured during the test. The machine vibrations were measured for using a piezo film ACH-01-03 accelerometer and digitized at $12.8 \mathrm{kHz}$ by an embedded acquisition platform. In each measurement 20 seconds of data was acquired. The acquisition platform consists of a Beaglebone Black single board computer with a Linux operating system, supplemented with a customized 6 channel interface. This embedded platform is used as a compact, open, scalable and cost-effective data acquisition system.

The accelerated life time test was performed on a FAG 6205 ball bearing. Before the start of the test a small indentation with a diameter of $230 \mu \mathrm{m}$ was created in the inner race using a Rockwell $\mathrm{C}$ hardness tester. This indentation is used as a local stress riser and represents a local plastic deformation caused by, for instance, a contamination particle. Subsequently, the accelerated life time test was performed for several hours. Although bearings can fail in many different ways, the indentation triggers the bearing to fail in a more repeatable way. The test was stopped when severe rolling contact surface fatigue occurred at the inner race (Halme \& Andersson, 2009). The start and the end condition of the inner race of the test bearing are shown in Figure 5.

Only a single dataset has been used in this paper for the purpose of comparing the three methods. However, the accelerated lifetime test has been performed several times as part of other research by authors. They have all resulted in similar surface fatigue faults at the inner race of the test bearing.
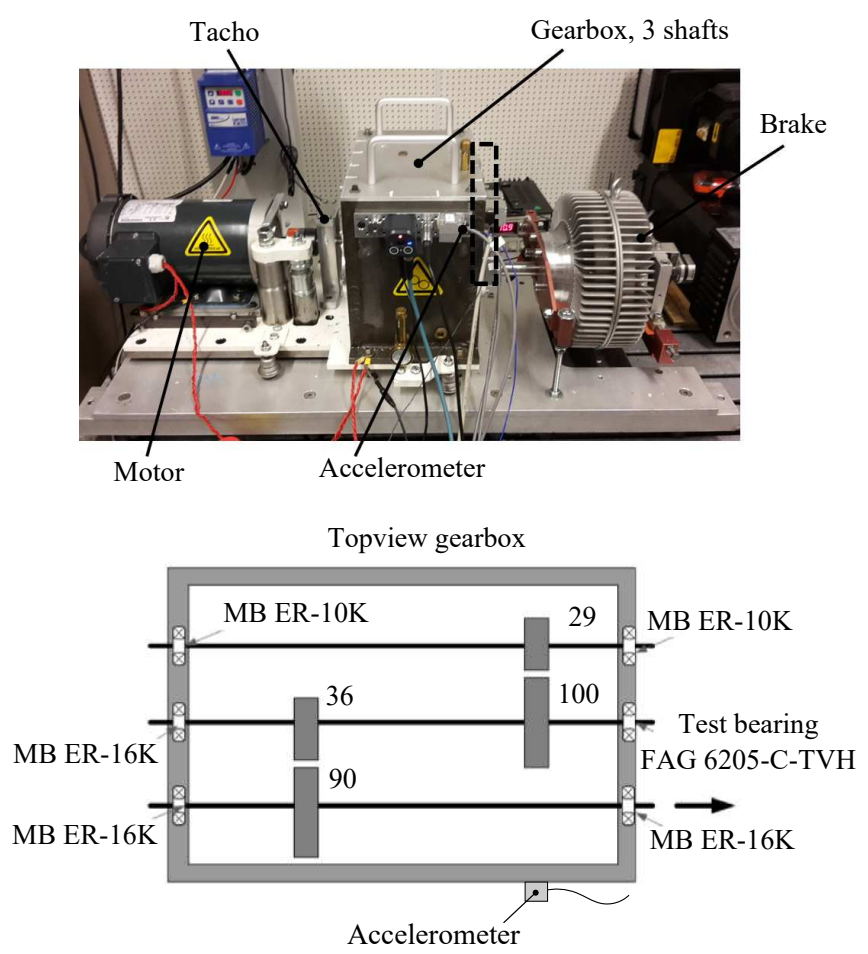

Figure 6. Gearbox set-up comprising a motor, 3-shaft gearbox and brake to introduce a load.

\subsection{Gearbox test}

The second test performed in this benchmark study was an industrially representative gearbox setup. Figure 6 shows a photograph and a schematic top-view of the gearbox setup. The test setup consists of (i) an induction electric motor, (ii) a gearbox and (iii) a magnetic brake. The motor is controlled by a variable-frequency-drive (VFD) with either a stationary mode or a transient mode (run-up/run-down). The motor speed can be controlled from 0 to $3000 \mathrm{rpm}$. The gearbox input shaft is connected to the motor through a flexible coupling, while the gearbox output shaft is directly coupled to the brake. The torque applied to the brake can be adjusted by the controller from 0 to $50 \mathrm{Nm}$.

As illustrated in Figure 6, the gearbox comprises of threeparallel shafts connected through contacting spur gear pairs. Note that the number of gear teeth is indicated in the figure. Hence the total reduction factor from the input to the output shaft is equal to $(100 / 29) \times(90 / 36)=8.62$. The input shaft is supported by deep groove ball bearings MB ER-10K, while the other shafts are supported by deep groove ball bearings MB ER-16K. For simulating a healthy or faulty state on the gearbox, the right-side bearing housing that supports the second shaft is equipped either with a healthy or a damaged ball bearing of type FAG 6205-C-TVH. The theoretical fault frequencies of this bearing of interest are listed in Table 1.

Two healthy bearings and three faulty bearings with different 


\begin{tabular}{l|ll}
\hline Abbreviation & Fault & $\begin{array}{l}\text { Frequency } \\
{[\mathbf{H z}]}\end{array}$ \\
\hline SHFT & Shaft speed & 1.000 \\
\hline BPFI & Inner race defect & 5.415 \\
\hline BPFO & Outer race & 3.585 \\
\hline FTF & Cage defect & 0.398 \\
\hline BSF & Ball spin & 2.358 \\
\hline BDF & Rolling element defect & 4.715 \\
\hline
\end{tabular}

Table 1. Theoretical bearing fault frequencies of a $6205-\mathrm{C}-$ TVH ball bearing at a shaft speed of $60 \mathrm{rpm}$.

inner race faults were tested. An indentation fault with a diameter of $490 \mu \mathrm{m}$ was created using an Rockwell C hardness tester. Two other bearings with operational faults were created using the accelerated life time test setup as described in Section 3.1. The healthy bearings are referred as 'Healthy1' and 'Healthy2', while the faulty bearings are referred as 'Indent', 'Faulty1', 'Faulty2', in the order of increasing severity and are illustrated in Figure 7.

For each healthy or faulty state, 3 operating conditions were imposed on the gearbox setup, namely three different motor speeds of 600, 1500 and $3000 \mathrm{rpm}$. The brake torque was kept constant at $50 \mathrm{Nm}$. Because of the transmission ratio, the rotational speed of the second shaft is 29/100 lower than that of the motor speed, while the torque applied on the second shaft is $36 / 90$ lower than that of the brake torque. Hence, for the imposed operating conditions, the rotational speeds of the second shaft were 174, 435 and $870 \mathrm{rpm}$, while the torque applied to the second shaft was $20 \mathrm{Nm}$. A high-end PCB accelerometer was mounted on the gearbox housing as shown in Figure 6. The vibration signals were sampled at $50 \mathrm{kHz}$ using a Dewesoft data acquisition system. For each operating condition, $10 \times 20$ seconds were repeated. The data are then processed using scripts written in Matlab.

\section{RESUlts}

The results obtained by the three methods are presented in this section. The accelerated life time test results are addressed first. This is followed by the results for gearbox test.

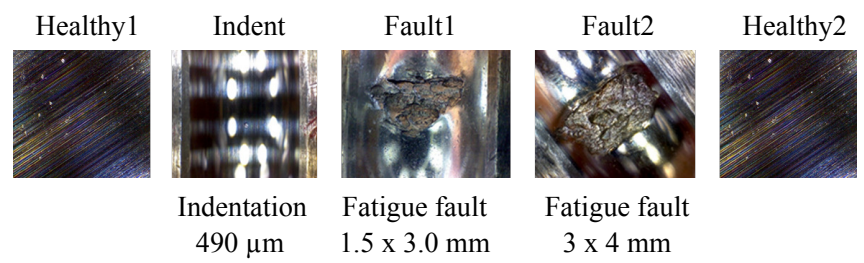

Figure 7. Five bearing states tested on the gearbox setup comprising two healthy bearings and three faulty bearings with different severities.
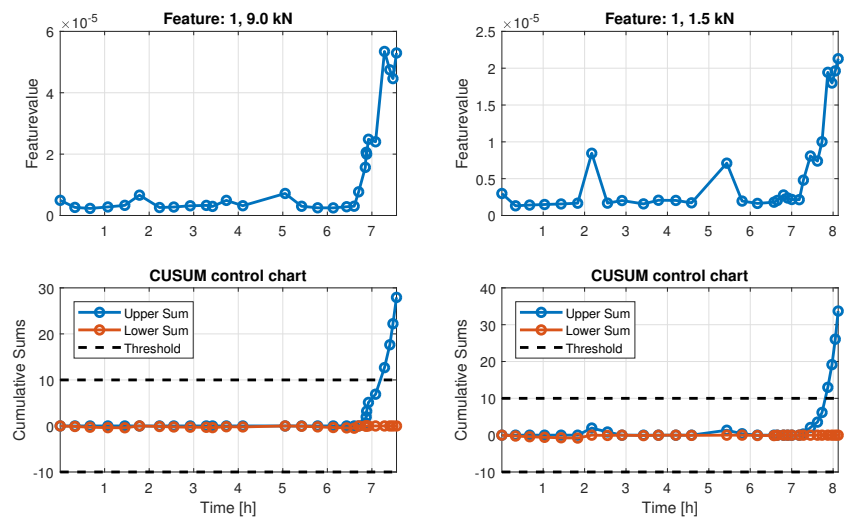

Figure 8. Feature values and CUSUM control charts for feature 1 in the ALT test for load $9.0 \mathrm{kN}$ (left) and $1.5 \mathrm{kN}$ (right).

\subsection{Accelerated life time test}

\subsubsection{Results method LCM}

Based on the raw sensor signals, the features were extracted in an overlapping sliding window approach. Using a window length of 0.2 seconds and an overlap of 0.1 seconds, we extracted 199 observations out of each 20 second data batch. However, for final evaluation, we observed only the mean value of those 199 observations for a data batch. Unlike the gearbox setup, we had no data of different health states available for feature selection and classifier training in the accelerated life time test. Therefore we were restricted to detect significant changes in the feature values. For that purpose, cumulative sum (CUSUM) control charts (Hawkins \& Olwell, 1998) were applied to the features.

Due to the missing feature selection step, we evaluated the top ranked features of the $3000 \mathrm{rpm}$ motor speed gearbox test (see Section 4.2.1). All of those features increased significantly towards the end of the test run for the $9.0 \mathrm{kN}$ as well as for the $1.5 \mathrm{kN}$ load conditions. For example, feature 1 is depicted in Figure 8. Due to the increasing feature value, the upper threshold of the CUSUM control chart was exceeded after approximately 7.3 hours in the $9.0 \mathrm{kN}$ case and 7.9 hours in the $1.5 \mathrm{kN}$ case, indicating a failure of the bearing.

For control reasons, we also show an arbitrarily chosen features here (feature 57 in Figure 9). The features showed no significant trend, and the CUSUM control charts didn't exceed the thresholds.

\subsubsection{Results method IMS}

In the accelerated life time test, only healthy data were available at the beginning. Thus the health assessment approach was applied to estimate the health state of the bearing during the whole testing process (see Section 2.2). Since the ALT was employed under two loads, the health model was constructed for $9.0 \mathrm{kN}$ and $1.5 \mathrm{kN}$ respectively. Vibration data 

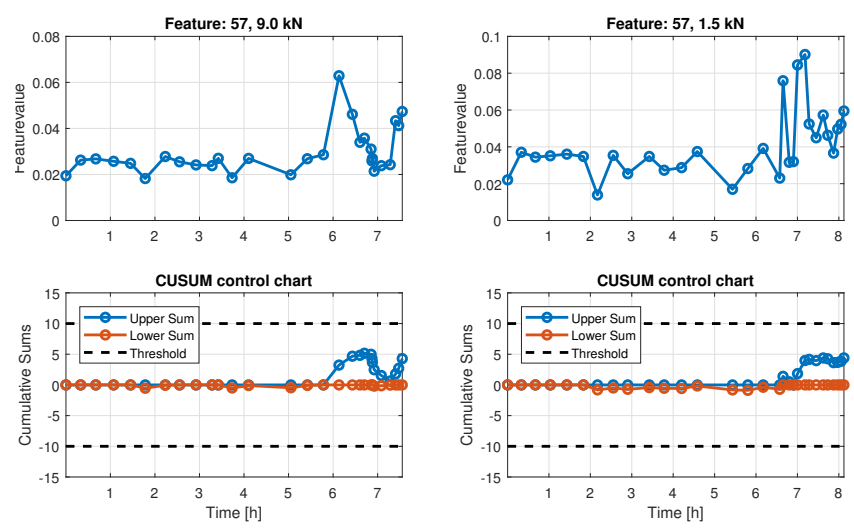

Figure 9. Feature values and CUSUM control charts for feature 57 in the ALT test for load $9.0 \mathrm{kN}$ (left) and $1.5 \mathrm{kN}$ (right)

were processed for each observation and the first 10 samples were utilized to train the baseline model. The health state was then represented by the assessment score, which was denoted by the MQE value, in the testing stage.

The health assessment results are shown in Figure 10 and Figure 11. Figure 10 depicts the assessment scores for the bearing under $9.0 \mathrm{kN}$ while Figure 11 represents the assessment scores under $1.5 \mathrm{kN}$. For the case of $9.0 \mathrm{kN}$, the assessment score varies at a low level for approximate 6 hours and then it increases significantly until the end of the bearing life. Since larger assessment score indicates farther distance between the current behavior and the baseline model, it is demonstrated that the health assessment approach is able to estimate the degradation process during the accelerated life time test. Further, we calculated the contribution of each feature to the MQE assessment score, which is illustrated in Figure 10 right. The feature with the largest contribution to assessment result is the ball pass frequency inner (BPFI), which is an inner race fault related signature. Since the accelerated life time test resulted in an inner race fault, it demonstrates that the method could also detect the location of the bearing fault.

Similar to the $9.0 \mathrm{kN}$ load case, the MQE assessment score also gradually increases after approximately 6.5 hour for the $1.5 \mathrm{kN}$ load case. However, the increase is slightly slower than that for the $9.0 \mathrm{kN}$ load case. The feature contribution (see Figure 11 right) again shows that the degradation was mainly driven by the inner race fault. The contribution of the inner race fault feature (i.e. BPFI) loses its weight although it is still dominant among all features. It reveals that under a lower load, it is more difficult to detect the degradation at an early stage.

\subsubsection{Results method FM}

The algorithm described in Section 2.3 was applied to the raw acceleration signal acquired at the $1.5 \mathrm{kN}$ and $9.0 \mathrm{kN}$
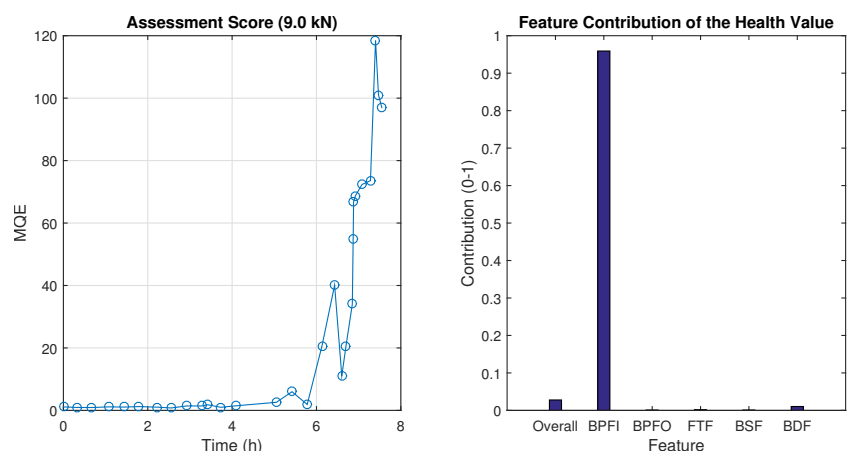

Figure 10. Health assessment results for load $9.0 \mathrm{kN}$, with the evolution of the MQE assessment score on the left and an overview of the contribution of the individual features on the right.
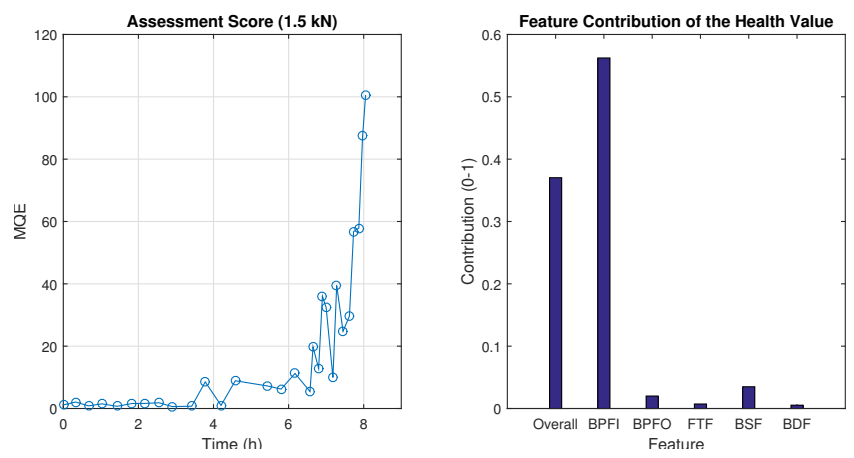

Figure 11. Health assessment results for load $1.5 \mathrm{kN}$, with the evolution of the MQE assessment score on the left and an overview of the contribution of the individual features on the right.

radial load condition. For each measurement 5 seconds of the acceleration signal was processed.

Figure 12 shows the evolution of five bearing features as function of the test time for the $1.5 \mathrm{kN}$ case. The features are defined in correspondence to Table 1. During the first few hours, no significant change in any of the feature value is observed. The inner race fault bearing feature (i.e. BPFI) starts to increase after approximately 6.7 hours in the test and significantly increases towards the end of the test. The other features are barely affected by the degradation process. This indicates the unique detection of an inner race fault at the test bearing.

Similarly, the acceleration signals acquired during the measurements at $9.0 \mathrm{kN}$ have also been analyzed separately. The evolution of the feature values are shown in 13. In contrast to the measurements at $1.5 \mathrm{kN}$, an increased inner race fault feature has been observed after only 6.1 hours. The increased loading condition has resulted into a slightly earlier detection of the bearing degradation. 


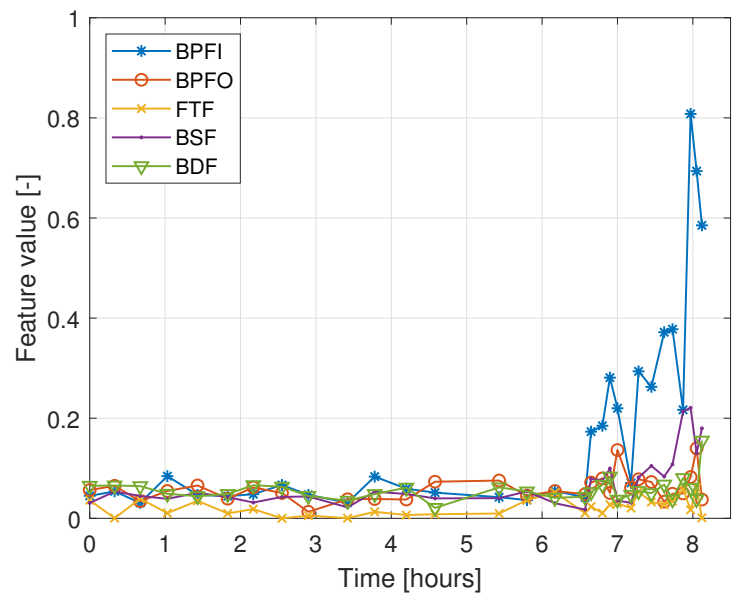

Figure 12. The evolution of five bearing fault features during the accelerated life time test of the ball bearing for all measurements at $1.5 \mathrm{kN}$ radial load.

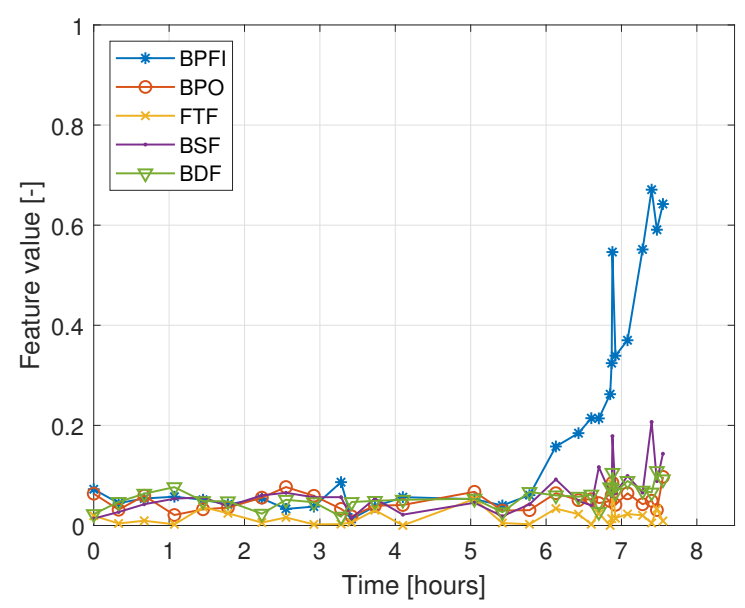

Figure 13. Evolution of five bearing fault features during the accelerated life time test of the ball bearing for all measurements at $9.0 \mathrm{kN}$ radial load.

\subsection{Gearbox test}

The results obtained by the three algorithms applied to the gearbox data are described in this section.

\subsubsection{Results method LCM}

Just like in the accelerated life time test, the features were extracted from the raw acceleration signals in an overlapping sliding window approach with a window length of $0.2 \mathrm{sec}-$ onds and an overlap of 0.1 seconds. This procedure resulted again in 199 observations for each 20 second data batch. After extracting all features, the feature selection algorithm was applied to the $3000 \mathrm{rpm}, 1500 \mathrm{rpm}$ and $600 \mathrm{rpm}$ motor speed data independently. Furthermore, we did not use all available states for feature selection. We used only the datasets

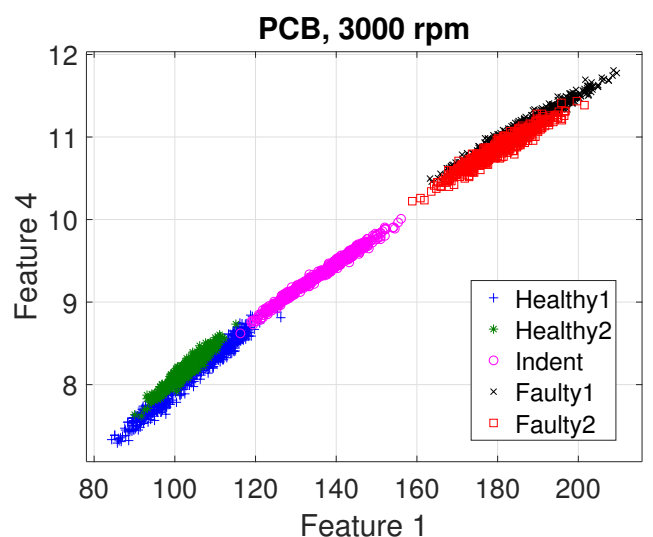

Figure 14. Scatter plot of top 2 features for the $3000 \mathrm{rpm}$ dataset.

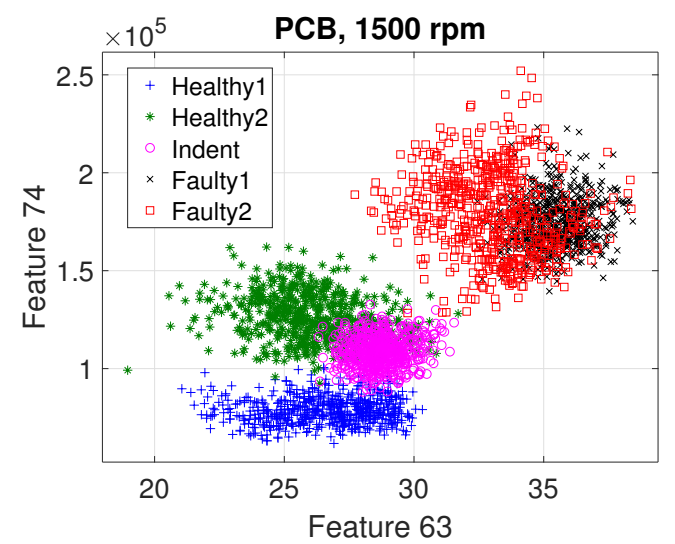

Figure 15. Scatter plot of top 2 features for the $1500 \mathrm{rpm}$ dataset.

of the states Healthy1, Healthy2 and Faulty2, but not Indent and Faulty1. For the 3000 rpm motor speed data, the algorithm selected 3 top ranked features, for the $1500 \mathrm{rpm}$ data it selected 4 top ranked features, and for the $600 \mathrm{rpm}$ data it selected 2 top ranked features. However, for a first visual impression we show only the top 2 features for all recorded rotation speeds and all recorded states in a scatter plot in Figure 14 (3000 rpm motor speed), Figure 15 (1500 rpm) and Figure 16 (600 rpm).

We can already notice a few things by just looking at those scatter plots:

- Different top features were selected for the different rotation speeds.

- The $3000 \mathrm{rpm}$ dataset revealed the best separability.

- Faulty1 and Faulty2 produced similar features.

- In the $3000 \mathrm{rpm}$ dataset, the Indent-class lies somewhere in-between the healthy and the faulty states.

For classification accuracy estimation, we trained a classifier only with the states Healthy1, Indent and Faulty2 (using 


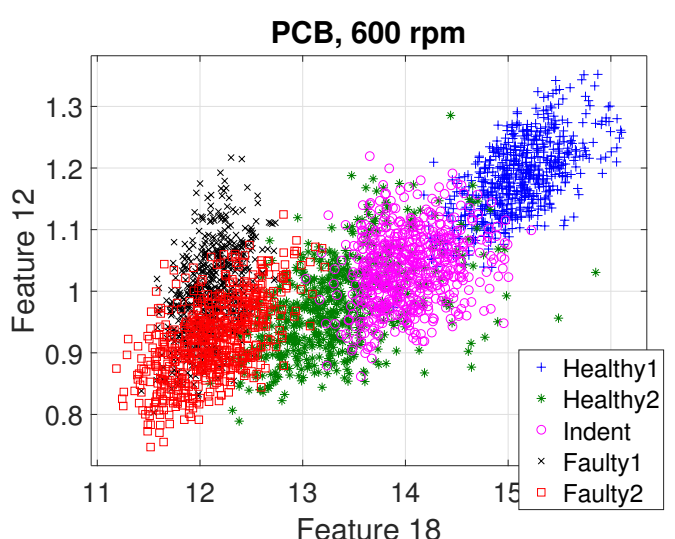

Figure 16. Scatter plot of top 2 features for the $600 \mathrm{rpm}$ dataset.

\begin{tabular}{l|l|l|l|l}
\hline \multicolumn{2}{c|}{} & \multicolumn{3}{|l}{ Estimated State } \\
\cline { 3 - 5 } \multicolumn{2}{c|}{} & Healthy & Indent & Faulty \\
\hline \multirow{4}{*}{\begin{tabular}{l} 
True State \\
\cline { 2 - 4 }
\end{tabular}} & Healthy1 & 177 & 22 & 0 \\
\cline { 2 - 5 } & Healthy2 & 199 & 0 & 0 \\
\cline { 2 - 5 } & Indent & 0 & 199 & 0 \\
\cline { 2 - 5 } & Faulty1 & 0 & 0 & 199 \\
\cline { 2 - 5 } & Faulty2 & 0 & 0 & 199 \\
\hline
\end{tabular}

Table 2. Confusion matrix for $3000 \mathrm{rpm}$ data.

two 20 second data batches each). After training, validation was performed with another 20 second data batch of all 5 bearing states. However, as target class of the classifier we did not use those five states, but only the simplified states Healthy (Healthy1 and Healthy2), Indent and Faulty (Faulty1 and Faulty2). According to the feature selection step, the $3000 \mathrm{rpm}$ data were validated with the top 3 features, the 1500 rpm data were validated with the top 4 features and the 600 rpm data were validated with the top 2 features. The classification accuracy for the $3000 \mathrm{rpm}$ data was $97.7 \%$ (confusion matrix in Table 2), for the $1500 \mathrm{rpm}$ data $77.3 \%$ (confusion matrix in Table 3) and for the $600 \mathrm{rpm}$ data $79.8 \%$ (confusion matrix in Table 4). That confirms the first visual impression: in the $3000 \mathrm{rpm}$ case, the separability of the states Healthy, Indent and Faulty in the feature space is satisfying, while the separability is worse in the $1500 \mathrm{rpm}$ and in the $600 \mathrm{rpm}$ case. Especially the state Healthy2 is misclassified in the $1500 \mathrm{rpm}$ and $600 \mathrm{rpm}$ case. As can be seen in the according scatter plots, the state Healthy 2 overlaps with the states Indent and Faulty. Since Healthy 2 was not used to train the classifier it is obvious that it will be misclassified.

\subsubsection{Results method IMS}

The fault diagnosis approach was applied to all gearbox data sets including 3 rotating speeds: $600 \mathrm{rpm}, 1500 \mathrm{rpm}$, and $3000 \mathrm{rpm}$. In the feature extraction step, instead of calculating features by a sliding window, which was mentioned in

\begin{tabular}{l|l|l|l|l}
\hline \multicolumn{2}{c|}{} & \multicolumn{3}{|l}{ Estimated State } \\
\cline { 3 - 5 } \multicolumn{1}{c|}{} & Healthy & Indent & Faulty \\
\hline \multirow{4}{*}{ True State } & Healthy1 & 173 & 26 & 0 \\
\cline { 2 - 5 } & Healthy2 & 0 & 102 & 97 \\
\cline { 2 - 5 } & Indent & 0 & 198 & 1 \\
\cline { 2 - 5 } & Faulty1 & 0 & 0 & 199 \\
\cline { 2 - 5 } & Faulty2 & 0 & 0 & 199 \\
\hline
\end{tabular}

Table 3. Confusion matrix for $1500 \mathrm{rpm}$ data.

\begin{tabular}{l|l|l|l|l}
\hline \multicolumn{2}{c|}{} & \multicolumn{3}{|l}{ Estimated State } \\
\cline { 3 - 5 } \multicolumn{1}{c|}{} & Healthy & Indent & Faulty \\
\hline \multirow{4}{*}{ True State } & Healthy1 & 199 & 0 & 0 \\
\cline { 2 - 5 } & Healthy2 & 2 & 86 & 111 \\
\cline { 2 - 5 } & Indent & 1 & 195 & 3 \\
\cline { 2 - 5 } & Faulty1 & 0 & 0 & 199 \\
\cline { 2 - 5 } & Faulty2 & 0 & 0 & 199 \\
\hline
\end{tabular}

Table 4. Confusion matrix for $600 \mathrm{rpm}$ data.

section 4.2.1, fault signatures were extracted from the whole vibration signal for each observation. Once the feature matrix were prepared, 15 samples for each health state were selected respectively to train the classification model. The other samples were utilized to test it. In the training stage, we trained two types of healthy data separately since they were measured temporally differently. The confusion matrix was selected as the evaluation metric. The diagnostic results generated by the machine learning model were compared with the ground truth from the experiments.

The fault diagnosis results were summarized in Figure 17, Figure 18, and Figure 19. In these figures, 'Target Class' denotes the ground truth while 'Output Class' represents the diagnosis results from the SOM model. Number 1 to 5 represent the five health conditions: Healthy1, Healthy2, Indent, Faulty1, and Faulty2. In $600 \mathrm{rpm}$ rotating speed case, which is shown in Figure 17, almost all testing data are diagnosed correctly. Only one Healthy2 sample is misclassified as Healthy1. The overall accuracy (96.2\%) validates that even under relatively low rotating speed the proposed method enables satisfied classification capabilities. In addition, similar diagnosis results are obtained in $1500 \mathrm{rpm}$ case (90.6\% accuracy) and $3000 \mathrm{rpm}$ case (93.9\% accuracy), which are illustrated in Figure 18 and Figure 19 respectively. Compared with 600 rpm case, however, there are misclassified samples for Indent, Faulty 1, and Faulty 2 failure modes in 1500 rpm and 3000 rpm cases.

Further, the contribution of each feature to the diagnosis result was investigated. Two examples are illustrated in Figure 20 and Figure 21. Figure 20 shows the feature contributions for the $600 \mathrm{rpm}$ case with the indent fault, while Figure 21 indicates the feature contribution for the $3000 \mathrm{rpm}$ case with the most severe fault, i.e. Faulty2. When the rotating speed is 


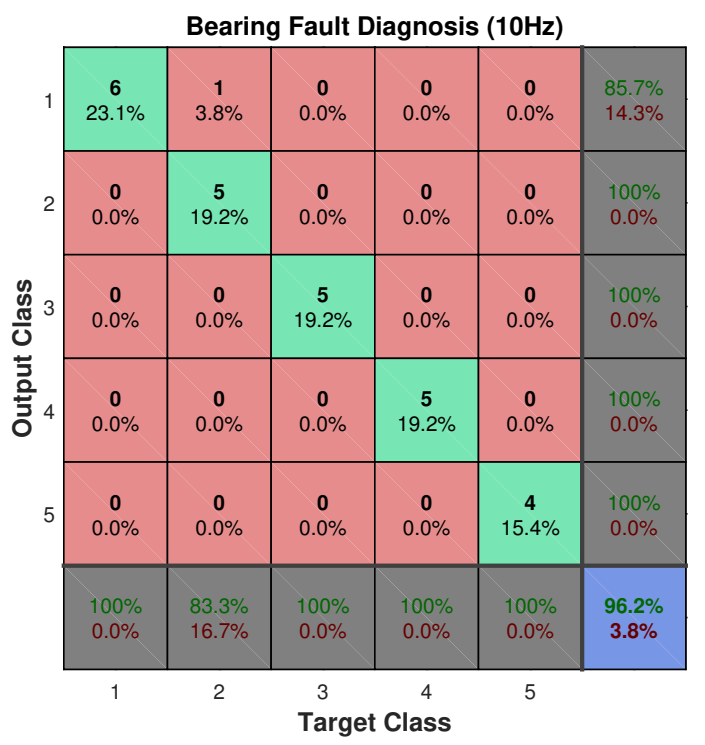

Figure 17. Confusion matrix for $600 \mathrm{rpm}$ data. The target class indicates the true bearing state, the output class refers to the estimated bearing state.

low, the feature representing the overall vibration level rather than bearing specific features contribute to the diagnosis results. While bearing specific features (i.e. BPFI) demonstrate to have more dominant contributions under higher rotating speed. Therefore, as the rotating speed increases and the bearing fault propagates, the proposed method by IMS is able to better perform the fault diagnosis.

Summarizing, under various rotating speeds, the proposed fault diagnosis approach was demonstrated to identify bearing faults and classify them based on different degradation levels.

\subsubsection{Results method FM}

The acquired data were processed using the algorithm described in section 2.3. For each bearing state and for each motor speed, 20 acceleration signals with a length of 5 seconds were analyzed. The feature values were computed and the threshold was defined using only the data of the 'Healthy1' measurement.

The distributions of the BPFI feature in case of a $3000 \mathrm{rpm}$, $1500 \mathrm{rpm}$ and $600 \mathrm{rpm}$ motor speed are respectively shown in Figure 22, 23 and 24. The central mark indicates the median, the bottom and top edges of the box indicate the 25th and the 75th percentiles, respectively. The distances between the top and bottom are the interquartile ranges. Observations that are more than 1.5 times the interquartile range away from the top or bottom of the box are considered as an outlier and are indicated by a ' + ' sign. The whiskers are lines extending above

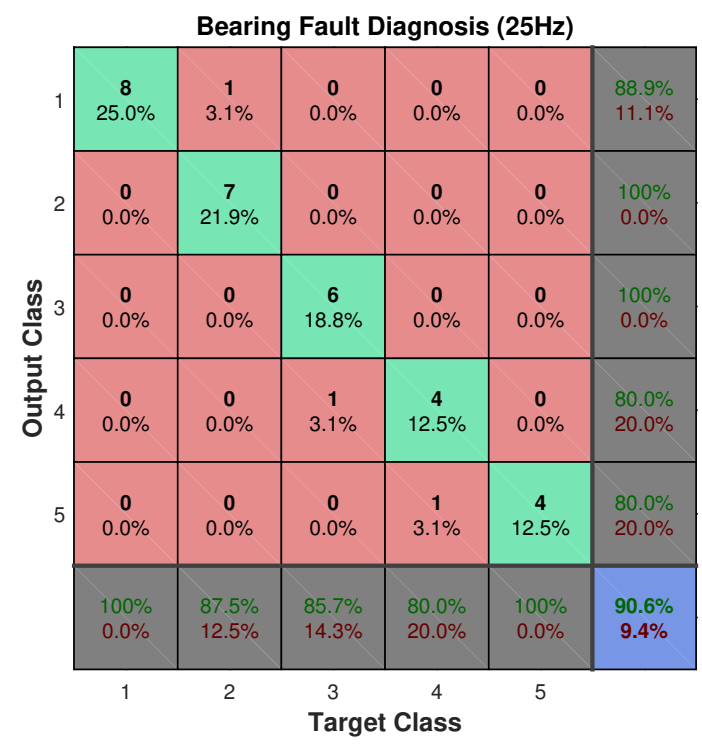

Figure 18. Confusion matrix for $1500 \mathrm{rpm}$ data. The target class indicates the true bearing state, the output class refers to the estimated bearing state.

and below each box to the most extreme data points that are not considered as outliers.

The results show that the algorithm can clearly discriminate between the Healthy 1 and the Faulty 2 state for the 3000 rpm and $1500 \mathrm{rpm}$ case. The difference in the extracted squared envelope spectra between Healthy 1 and Faulty 2 is also shown in Figure 25 for the $1500 \mathrm{rpm}$ case. It is also observed that the degree of separation between Healthy1 and Faulty2 is larger for the $1500 \mathrm{rpm}$ than for the $3000 \mathrm{rpm}$ case. This is caused by a system resonance frequency at around $1200 \mathrm{rpm}(20 \mathrm{~Hz})$. The Indent and Faulty 1 state are not detectable for either of the motor speeds. In case of the $600 \mathrm{rpm}$, none of the faulty states are detected.

It was expected that Healthy 1 and Healthy 2 would reveal similar feature values. However, the Healthy 2 bearing shows slightly higher feature values for all three rotational speeds, resulting in false positive classification. This effect is also shown in the associated squared envelope spectra. It is expected that those slightly higher values for Healthy 2 are caused by reported assembling issues of the test bearing in its gearbox housing during the benchmark test campaign.

\section{Discussion}

The three bearing diagnostic algorithms are compared based on their diagnostic performance, applicability, training and configuration requirements, level of automation in this section. The computational cost of each methods has not been compared since different computing hardware were used to 


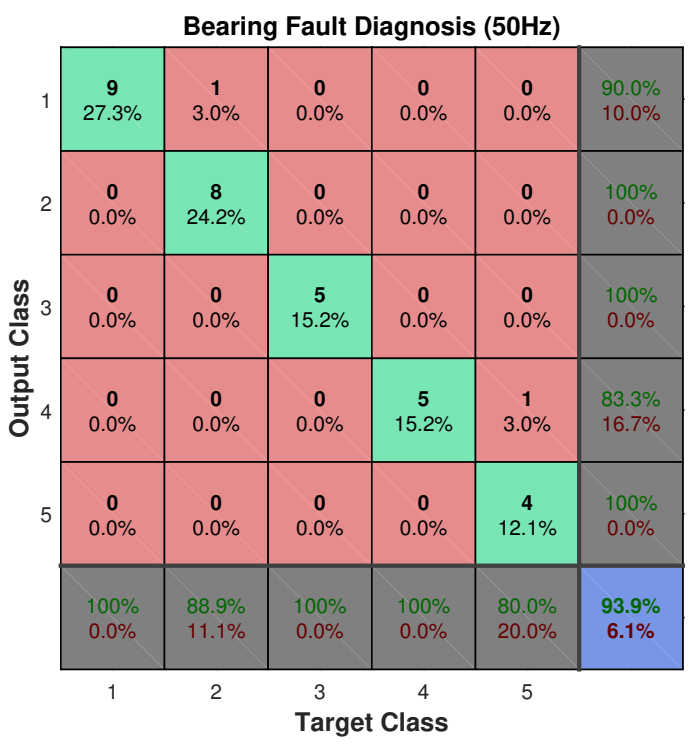

Figure 19. Confusion matrix for $3000 \mathrm{rpm}$ data. The target class indicates the true bearing state, the output class refers to the estimated bearing state.

calculate the results. Finally, the most important pros and cons of each individual method are summarized in Table 5 at the end of this section.

\subsection{Diagnostic performance}

All three methods were able to describe the evolution of the inner race bearing fault during the accelerated life time test. The methods developed by IMS and FM were able to detect the presence of the bearing degradation earlier, i.e. 6.7 hours, than the purely data driven method of LCM, i.e. 7.3 hours, under the $1.5 \mathrm{kN}$ nominal load condition. It reveals a slightly higher sensitivity of the methods of IMS and FM to detect incipient inner race bearing fault for this test. They were also able to determine the location of the bearing fault (i.e. inner race), using unique bearing fault features for each type of fault.

The differences are explained by the fact that each method made use of a different set of features that are sensitive to bearing faults. The method of IMS used a combination of a few statistical time domain features and bearing fault specific features. The method of LCM used a very wide set of statistical features. The method of FM only relies on the bearing fault specific features.

Although this paper is limited to the evaluation of the bearing current health condition, the accelerated life time test as described in section 3.1 can form a basis for prognostics and health management. Constructing the right health indicators is key in predicting the remaining useful life (D. Wang et al.,

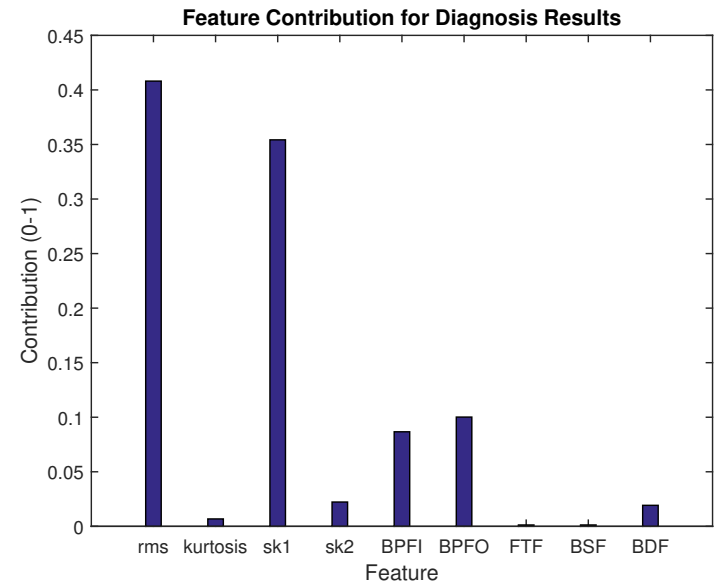

Figure 20. Contribution of all features for the diagnosis (600 rpm, Indent).

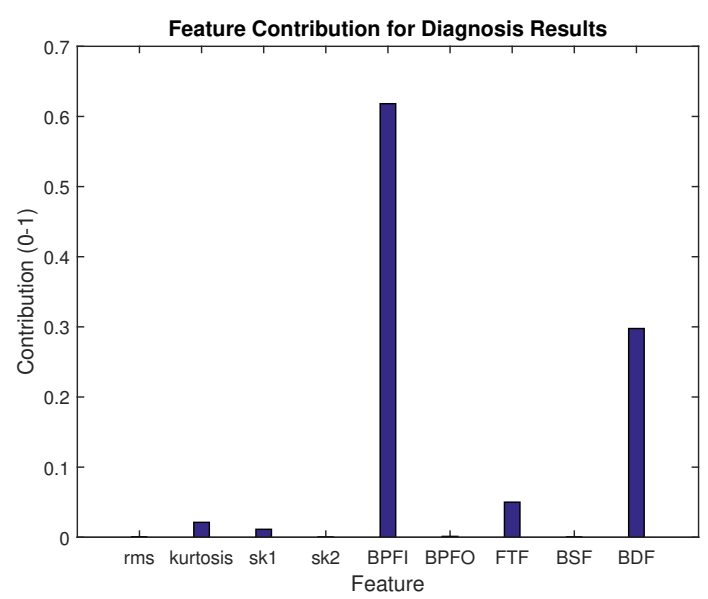

Figure 21. Contribution of all features for the diagnosis (3000 rpm, Faulty2).

2017). The accelerated life time test can be used to evaluate and validate remaining useful life estimation techniques. Ultimately this could serve planning the optimal maintenance strategy according to the systems current and future health condition and the costs.

In the gearbox test, different classification classes have been used in each method. The method of IMS used 4 classes, the method of LCM used 3 classes, the method of FM used a single threshold based on only healthy data to classify between the healthy and the faulty state.

Based on the classification results, the method of IMS and LCM seem to outperform the method of FM in the gearbox test, in particular for the smaller faults. The method of IMS and LCM were able to classify all the bearing faults with different levels of accuracy, whereas the method of FM only detected the most severe fault, i.e. Faulty2. By the contribution matrix in Figure 20 and Figure 21 IMS showed that the 


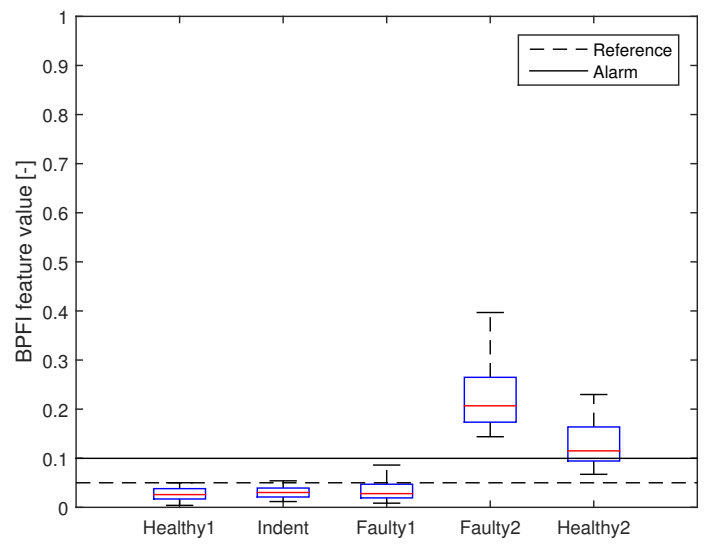

Figure 22. BPFI feature as function of the different bearing states at a motor speed of $3000 \mathrm{rpm}$, a shaft rotation speed of $870 \mathrm{rpm}$ of the test bearing.

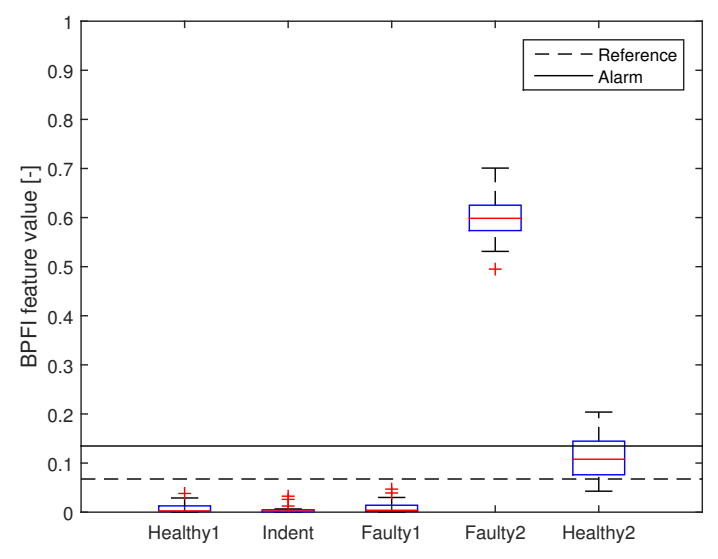

Figure 23. BPFI feature as function of the different bearing states at a motor speed of $1500 \mathrm{rpm}$, a shaft rotation speed of $435 \mathrm{rpm}$ of the test bearing.

smallest faults were to a large extend detected based on a statistical time domain feature rather than bearing fault specific features. While the bigger faults were primarily detected by the bearing specific features. This matches with the observations for the method of FM, which relies purely on bearing specific features.

The high classification accuracy obtained for the method of IMS and LCM can be explained by a high correlation between the training and testing data. Although the training and testing data were acquired in different measurements, they were collected in the same measurement campaign, for the same bearing sample and for the same bearing assembly. As a result, this could lead to a misleading classification accuracy. Firstly, because the training and testing data of one test bearing can be correlated not only in terms of the bearing faults,

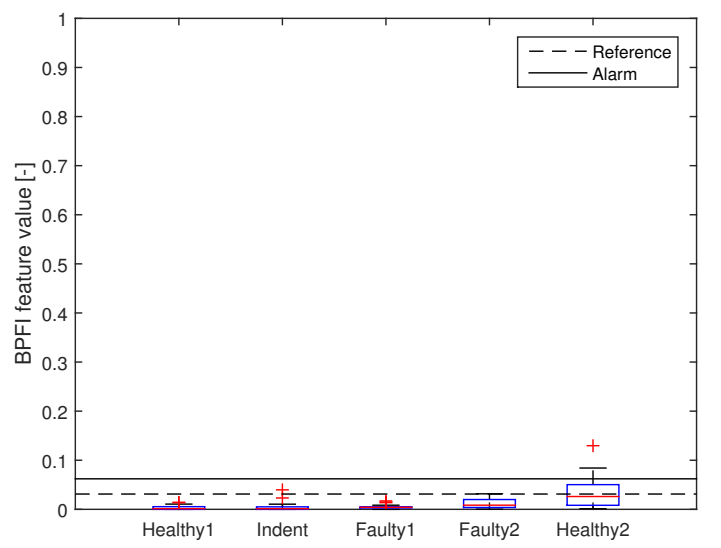

Figure 24. BPFI feature as function of the different bearing states at a motor speed of $600 \mathrm{rpm}$, a shaft rotation speed of $174 \mathrm{rpm}$ of the test bearing.

but also by other e.g. bearing assembly related effects. Secondly, the classification differences between various healthy and faulty states are not uniquely caused by the bearing fault, but could also be caused by e.g. bearing assembly differences. The classification results are therefore lacking in a setup wide validation. Attention should be paid to the quality of the training data. The training data should comprise all possible system variations, including for instance new installations of bearings and other bearing samples.

The method of IMS and FM both allows to indicate the location of the bearing fault based on a unique bearing feature for each fault. This requires prior knowledge of the setup and hence bearing specific fault frequencies, which is not required for the method of LCM.

\subsection{Practical applicability}

The method of IMS and FM are bearing specific monitoring solutions. They are not directly applicable to other type of monitoring problems. The method of LCM is on the other hand a widely applicable method for fault monitoring problems. It can be trained for most kind of mechanical failures, including bearing faults, as long as representative training data is available.

For the gearbox test, all methods show a reduced performance for the lowest rotational speed and have different levels of success for $1500 \mathrm{rpm}$ and $3000 \mathrm{rpm}$ cases. The method of IMS showed a high classification accuracy for $600 \mathrm{rpm}$ case due to a high contribution of statistical time domain features to the assessment score. The method of LCM showed the best separation between the classes for high rotation speeds. The method of FM performs slightly better for $1500 \mathrm{rpm}$ than for $3000 \mathrm{rpm}$ due to a system resonance frequency that is close to the rotational shaft speed leading to amplified vibration re- 

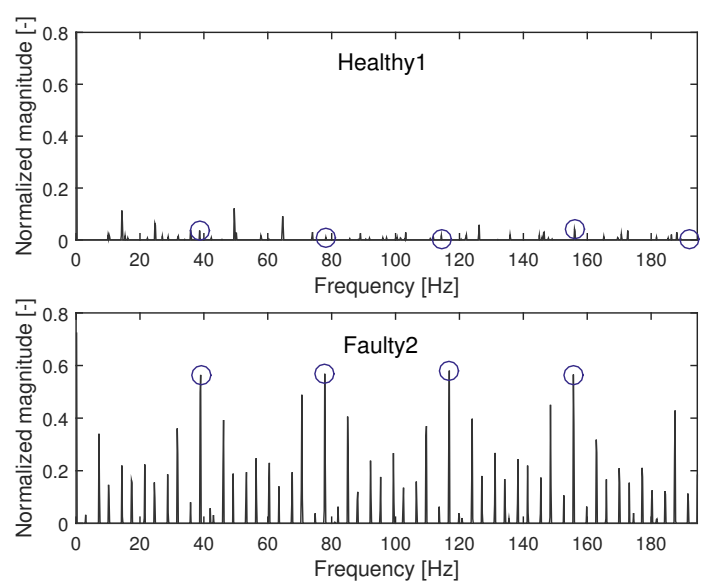

Figure 25. A comparison of the square envelop spectra of Healthy 1 and Faulty 2 test bearing at a 1500 rpm motor speed, showing the clear presence of BPFI related harmonics. The summed normalized magnitude of the first four harmonics indicated by the blue circles is used as a bearing faulty feature.

\section{sponses.}

The three methods have different levels of automation. The method of IMS is currently a semi-automated algorithm. Manual intervention is needed during the feature extraction process. The method can be fully automated further. The method of LCM is not yet automated. An automatic supervision by pre-defined feature sets is however possible. The feature set has to be selected manually in reference to the scenario. The method of FM is a fully automated algorithm and provides feature value in relation to the threshold value based on raw acceleration signals.

\subsection{Training and configuration requirements}

The method of LCM does not require any system knowledge, while the methods of IMS and FM do need system information. The information that is required in those algorithms comprise the (i) shaft rotational speed, (ii) bearing type or bearing geometry to calculate the bearing fault frequencies at the given speed and (iii) training data.

The performance of each method highly relies on the availability of validated training data. The methods of IMS and LCM need validated training data of both healthy and faulty bearing scenarios. The method of FM only relies on training data of healthy bearings. In practice the availability of training data of faulty bearings is typically scarce and could therefore make the adoption more complicated. Moreover, the type of bearing faults that can occur are very diverse, increasing the chances of misclassification in case of bearing monitoring. Once a fault occurs that is not part of training data, a proper detection and classification is not guaranteed.

The method of IMS used 7 minutes of training data for each fault state to train the SOM model. The method of LCM relied on 2 measurements of 20 seconds of each state to select the features and train the classifiers. The method of FM used 20 measurements of 5 seconds of vibration data at $50 \mathrm{kHz}$ to determine the detection threshold.

\section{CONCLUSION AND GUIDELINES}

Three vibration based condition monitoring algorithms for bearing fault detection have been compared based on their diagnostic performance, practical applicability (e.g. level of automation), training and configuration requirements.

Based on the accelerated life time test it is concluded that the method of IMS and FM, employing bearing specific features, showed to be more sensitive for early bearing fault detection than purely statistical features used in the method of LCM. In contrary, the method of LCM does not require specific system knowledge and is not limited to bearing condition monitoring only. The method is more generally and widely applicable to fault monitoring problems.

The methods of IMS and LCM seem to outperform the method of FM in the gearbox test, in particular for the smaller faults. However, the training and testing data used by those methods were acquired for the same bearing sample and for the same bearing assembly. This could lead to a high correlation between the training and testing data and hence a misleading classification accuracy. Therefore, attention should be paid to the quality of the training data. It is concluded that the training data should comprise all relevant system variations, including for instance remounting of the bearing. Otherwise, the training and testing data can be correlated not only in terms of bearing faults, but also due to other effects. Moreover, the classification differences between various healthy and faulty states is in that case not uniquely caused by the bearing fault, but could also be caused by those other effects.

The methods of IMS and LCM need validated training data of both healthy and all faulty bearing scenarios, whereas the method of FM relies on training data of healthy bearings only. In practice, the type of bearing faults that can occur is very diverse and the availability of training data of faulty bearings is typically scarce and could therefore make the adoption more complicated.

The findings of the benchmark study presented in this paper serve as a guideline for the selection of an appropriate method for bearing fault diagnosis.

\section{ACKNOWLEDGMENT}

The benchmark study described in this paper has been performed as part of the Mechatronic Alliance, an alliance consisting of Linz Center of Mechatronics (Austria), Flanders Make (Belgium), FIMECC (Finland) and E-TIME institute (France). 


\begin{tabular}{|c|c|c|}
\hline & Pros & Cons \\
\hline Method IMS & $\begin{array}{l}\text { - Method uses bearing specific features which } \\
\text { showed to be more sensitive to early fault } \\
\text { detection than purely statistical features. } \\
\text { - } \quad \text { Allows for multi-class classification. } \\
\text { - Can identify the fault location (e.g. inner } \\
\text { race, outer race). } \\
\text { - Combines a set of features to enhance detec- } \\
\text { tion performance. } \\
\text { - Semi-automated method. }\end{array}$ & $\begin{array}{l}\text { - Requires training data of both healthy and } \\
\text { faulty bearings for all scenarios. } \\
\text { - Requires specific system knowledge (e.g. } \\
\text { bearing geometry, rotational speed). } \\
\text { - Requires significant amount of training data } \\
\text { to train the self-organizing map (SOM). } \\
\text { - Limited to bearing monitoring only. }\end{array}$ \\
\hline Method LCM & $\begin{array}{l}\text { - Does not require specific system knowledge } \\
\text { (e.g. bearing type, rotational speed). } \\
\text { - } \\
\text { - Mllows for multi-class classification. } \\
\text { tion problems, not limited to bearing moni- } \\
\text { toring. }\end{array}$ & $\begin{array}{l}\text { - Requires training data of both healthy and } \\
\text { faulty bearings for all scenarios. } \\
\text { - Lack of physical understanding of the se- } \\
\text { lected features. } \\
\text { - Not yet automated, requires to manually se- } \\
\text { lect the feature set. }\end{array}$ \\
\hline Method FM & $\begin{array}{l}\text { - Method uses bearing specific features which } \\
\text { showed to be more sensitive to early fault } \\
\text { detection than purely statistical features. } \\
\text { - Can identify the fault location (e.g. inner } \\
\text { race, outer race). } \\
\text { - Algorithm is fully automated. } \\
\text { - Requires only healthy data to define the clas- } \\
\text { sification threshold. }\end{array}$ & $\begin{array}{l}\text { - Requires specific system knowledge (e.g. } \\
\text { bearing geometry, rotational speed). } \\
\text { - Limited to binary classification, no fault } \\
\text { severity estimation. } \\
\text { - Limited to bearing monitoring only. }\end{array}$ \\
\hline
\end{tabular}

Table 5. A summary of the most important pros and cons observed for each individual bearing faulty diagnostic method.

This work has been supported by the COMET-K2 "Center for Symbiotic Mechatronics" of the Linz Center of Mechatronics (LCM) funded by the Austrian federal government and the federal state of Upper Austria.

This research was supported by Flanders Make, the strategic research centre for the manufacturing industry.

\section{REFERENCES}

Alattas, M., \& Basaleem, M. (2007). Statistical analysis of vibration signals for monitoring gear condition. Damascus Univ. Journal, 23(2), 67-92.

Albarbar, A., Mekid, S., Starr, A., \& Pietruszkiewicz, R. (2008). Suitability of MEMS accelerometers for condition monitoring: An experimental study. Sensors, 8(2), 784-799.

Antoni, J., \& Randall, R. (2006). The spectral kurtosis: application to the vibratory surveillance and diagnostics of rotating machines. Mechanical Systems and Signal Processing, 20, 308-331.

Assaad, B., Eltabach, M., \& Antoni, J. (2014). Vibration based condition monitoring of a multistage epicyclic gearbox in lifting cranes. Mechanical Systems and Signal Processing, 42, 351-367.

Bajric, R., Zuber, N., Skrimpas, G., \& Mijatovic, N. (2016). Feature extraction using discrete wavelet transform for gear fault diagnosis of wind turbine gearbox. Shock and Vibration, 2016.

Barbini, L., Ompusunggu, A., Hillis, A., du Bois, J., \& Bartic, A. (2017, jul). Phase editing as a signal pre-processing step for automated bearing fault detection. Mechanical Systems and Signal Processing, 91, 407-421.

Bechhoefer, E., \& Kingsley, M. (2009). A review of time synchronous average algorithms. In Annual conference of the prognostics and health management society, san diego, ca, sept (pp. 24-33).

Boldt, F., Rauber, T., \& Varejão, F. (2013). Feature extraction and selection for automatic fault diagnosis of rotating machinery..

Borghesani, P., Pennacchi, P., Randall, R. B., Sawalhi, N., \& Ricci, R. (2013). Application of cepstrum prewhitening for the diagnosis of bearing faults under variable speed conditions. Mechanical Systems and Signal Processing, 36(2), 370-384.

Decker, H., \& Lewicki, D. (2003). Spiral bevel pinion crack detection in a helicopter gearbox. In Proceedings of the 59th annual forum and technology display.

de Ridder, D., et al. (Eds.). (2017). Classification, parameter estimation and state estimation: An engineering approach using matlab, 2nd edition. John Wiley \& Sons, Ltd.

Di, Y., Jin, C., Bagheri, B., Shi, Z., Ardakani, H., Tang, Z., \& Lee, J. (2018). Fault prediction of power electronics 
modules and systems under complex working conditions. Computers in Industry, 97, 1-9.

Dy, J., \& Brodley, C. (2004). Feature selection for unsupervised learning. Journal of Machine Learning Research, 5.

Guyon, I., \& Elisseeff, A. (2003). An introduction to variable and feature selection. Journal of Machine Learning Research, 3, 1157-1182.

Halme, J., \& Andersson, P. (2009). Rolling contact fatigue and wear fundamentals for rolling bearing diagnostics state of the art. Journal of Engineering Tribology, 224, 377-393.

Hawkins, D., \& Olwell, D. (Eds.). (1998). Cumulative sum charts and charting for quality improvement. Springer.

Heidari Bafroui, H., \& Ohadi, A. (2014). Application of wavelet energyband shannon entropy for feature extraction in gearbox fault detection under varying speed conditions. Neurocomputing, 133, 437-445.

Henriquez, P., Alonso, J., Ferrer, M., \& Travieso, C. (2014). Review of automatic fault diagnosis systems using audio and vibration signals. IEEE Transactions on Systems, Man, and Cybernetics: Systems, 44(5), 642-652.

Jablonski, A., Barszcz, T., Bielecka, M., \& Breuhaus, P. (2013). Modeling of probability distribution functions for automatic threshold calculation in condition monitoring systems. Measurement: Journal of the International Measurement Confederation, 46(1), 727-738. doi: 10.1016/j.measurement.2012.09.011

Jafarizadeh, M., Hassannejad, R., Ettefagh, M., \& Chitsaz, S. (2008). Asynchronous input gear damage diagnosis using time averaging and wavelet filtering. Mechanical Systems and Signal Processing, 22, 172-201.

Jalil, M., Butt, F. A., \& Malik, A. (2013). Short-time energy, magnitude, zero crossing rate and autocorrelation measurement for discriminating voiced and unvoiced segments of speech signals. In The international conference on technological advances in electrical, electronics and computer engineering (p. 208-212).

Jia, X., Zhao, M., Di, Y., Jin, C., \& Lee, J. (2017). Investigation on the kurtosis filter and the derivation of convolutional sparse filter for impulsive signature enhancement. Journal of Sound and Vibration, 386, 433-448.

Jia, X., Zhao, M., Di, Y., Li, P., \& Lee, J. (2018). Sparse filtering with the generalized lp/lq norm and its applications to the condition monitoring of rotating machinery. Mechanical Systems and Signal Processing, 102, 198-213.

Kohonen, T. (1998). The self-organizing map. Neurocomputing, 21(1-3), 1-6.

Kollialil, E. S., Gopan, K. G., Harsha, A., \& Joseph, L. A. (2013, Oct). Single feature-based non-convulsive epileptic seizure detection using multi-class svm. In 2013 international conference on emerging trends in communication, control, signal processing and com- puting applications (c2spca) (p. 1-6).

Konstantin-Hansen, H., \& Herlufsen, H. (2010). Envelope and ceptstrum analyses for machinery fault identification. Sound \& Vibration, 10-12.

Lei, Y., He, Z., Zi, Y., \& Hu, Q. (2007). Fault diagnosis of rotating machinery based on multiple anfis combination with gas. Mechanical Systems and Signal Processing, 21, 2280-2294.

McClintic, K., Lebold, M., Maynard, K., Byington, C., \& Campbell, R. (2000). Residual and difference feature analysis with transitional gearbox data. In Proceedings of the 54th meeting of the society for machinery failure prevention technology.

McFadden, P., \& Toozhy, M. (2000). Applications of synchronous averaging to vibration monitoring of rolling element bearings. Mechanical Systems and Signal Processing, 14(6), 891-906.

McLachlan, G. (1999). Mahalanobis distance. Resonance, 4(6), 20-26.

Ompusunggu, A. P., Ooijevaar, T., Kilundu, B., \& Devos, S. (2018). Automated bearing fault diagnostics with cost-effective vibration sensor. In J. Mathew, C. Lim, \& L. Ma (Eds.), Asset intelligence through integration and interoperability and contemporary vibration engineering technologies. proceedings of the 12th world congress on engineering asset management (pp. 463472). Springer International Publishing.

Randall, R., \& Antoni, J. (2011, feb). Rolling element bearing diagnostics - A tutorial. Mechanical Systems and Signal Processing, 25(2), 485-520.

Rao, V. (2015). Spectral kurtosis theory: A review through simulations. Global Journal of Researches in Engineering: Electrical and Electronics Engineering, 15(6), 49-61.

Sait, A., \& Sharaf-Eldeen, Y. (2011). A Review of Gearbox Condition Monitoring Based on vibration Analysis Techniques Diagnostics and Prognostics. In T. Proulx (Ed.), Rotating machinery, structural health monitoring, shock and vibration, volume 5, proceedings of the 29th imac, a conference on structural dynamics, 2011 (pp. 359-374). Jacksonville, FL; United States: The Society for Experimental Mechanics, Inc.

Satyam, M., Sudhakara, R., \& Devy, C. (1994). Cepstrum analysis - an advanced technique in vibration analysis of defects in rotating machinery. Defence Science Journal, 44(1), 53-60.

Sawalhi, N., Randall, R. B., \& Forrester, D. (2014). Separation and enhancement of gear and bearing signals for the diagnosis of wind turbine transmission systems. Wind Energy, 17(5), 729-743.

Sharma, V., \& Parey, A. (2016). A review of gear fault diagnosis using various condition indicators. Procedia Engineering, 144, 253-263.

Shen, C., Wang, D., Kong, F., \& Tse, P. (2013). Fault diagno- 
sis of rotating machinery based on the statistical parameters of wavelet packet paving and a generic support vector regressive classifier. Measurement, 46, 15511564.

Singh, S., \& Vishwakarma, M. (2015). A review of vibration analysis techniques for rotating machines. International Journal of Engineering Research \& Technology, 4(03), 757-761.

Suma, S. A., \& Gurumurthy, K. S. (2010). Novel pitch extraction methods using average magnitude difference function (amdf) for lpc speech coders in noisy environ- ments. In 2010 2nd international conference on signal processing systems (Vol. 1, p. V1-636-V1-640).

Tax, D. (2001). One-class classification (Unpublished doctoral dissertation). Delft University of Technology.

Wang, D., Tsui, K., \& Mia, Q. (2017). Prognostics and health management: A review of vibration based bearing and gear health indicators. IEEE Access, 6, 665-676.

Wang, H., \& Chen, P. (2007). Fault diagnosis of centrifugal pump using symptom parameters in frequency domain. Agricultural Engineering International: the CIGR Ejournal, IX. 